Homology, Homotopy and Applications, vol.16(2), 2014, pp.231-237

\title{
ANNIHILATION OF COHOMOLOGY AND DECOMPOSITIONS OF DERIVED CATEGORIES
}

\author{
SRIKANTH B. IYENGAR AND RYO TAKAHASHI
}

(communicated by J. Daniel Christensen)

\begin{abstract}
It is proved that an element $r$ in the center of a coherent ring $\Lambda$ annihilates $\operatorname{Ext}_{\Lambda}^{n}(M, N)$, for some positive integer $n$ and all finitely presented $\Lambda$-modules $M$ and $N$, if and only if the bounded derived category of $\Lambda$ is an extension of the subcategory consisting of complexes annihilated by $r$ and those obtained as $n$-fold extensions of $\Lambda$. This has applications to finiteness of dimension of derived categories.
\end{abstract}

\section{Introduction}

Let $\Lambda$ be a right coherent ring, $\bmod \Lambda$ the category of finitely presented right $\Lambda$ modules, and $D^{\mathrm{b}}(\Lambda)$ its bounded derived category. The purpose of this note is to prove the result below that reveals a close link between the existence of uniform annihilators of Ext-modules, as modules over the center $\Lambda^{c}$ of $\Lambda$, and a kind of decomposition of the derived category. In the statement, $\mathcal{G}$ is the class of morphisms in $D^{\mathrm{b}}(\Lambda)$ that induce the zero map in cohomology, $r$ is an element in $\Lambda^{\mathrm{c}}$, and $\mathrm{D}^{\mathrm{b}}(\Lambda)_{r}$ consists of complexes $X$ with $r \operatorname{Ext}_{\Lambda}^{0}(X, X)=0$, while $\mathrm{C} \diamond \mathrm{D}$ is the subcategory of complexes obtained as extensions of complexes in $\mathrm{C}$ and $\mathrm{D}$; see 2.1 .

Theorem 1.1. Fix a non-negative integer $n$ and an element $r$ in $\Lambda^{c}$. The following conditions on $\mathrm{D}^{\mathrm{b}}(\Lambda)$ are equivalent.

(1) $r \mathcal{G}^{n}=0$;

(2) $\mathrm{D}^{\mathrm{b}}(\Lambda)=\mathrm{D}^{\mathrm{b}}(\Lambda)_{r} \diamond\{\Lambda\}^{n \diamond}$;

(3) $\mathrm{D}^{\mathrm{b}}(\Lambda)=\{\Lambda\}^{n \diamond} \diamond \mathrm{D}^{\mathrm{b}}(\Lambda)_{r}$.

When they hold, $r \operatorname{Ext}_{\Lambda}^{n}(\bmod \Lambda, \bmod \Lambda)=0$. Conversely, the latter condition gives $r^{3} \mathcal{G}^{2 n}=0$.

This result is a consequence of Theorem 2.10, which applies to abelian categories with enough projectives. In fact, the equivalence of conditions (1)-(3), and the proofs,

The first author was supported in part by NSF grant DMS-1201889.

The second author was supported in part by JSPS Grant-in-Aid for Young Scientists (B) 22740008, JSPS Grant-in-Aid for Scientific Research (C) 25400038 and JSPS Postdoctoral Fellowships for Research Abroad.

Received May 20, 2014, revised July 25, 2014; published on September 29, 2014.

2010 Mathematics Subject Classification: 16E30, 16E35, 18G25.

Key words and phrases: cohomology annihilator, derived category, projective class.

Article available at http://dx.doi.org/10.4310/HHA.2014.v16.n2.a12

Copyright (C) 2014, International Press. Permission to copy for private use granted. 
carry over verbatim to generating projective classes in triangulated categories, in the sense of Christensen [1]; with Ext as in Section 4 of op. cit., the entire statement carries over.

Here is one application (see Corollary 2.12) of the theorem above: If $r \in \Lambda^{c}$ is a non-zerodivisor on $\Lambda$ and satisfies $r \mathcal{G}^{n}=0$, then there is an inequality

$$
\operatorname{dim} \mathrm{D}^{\mathrm{b}}(\Lambda) \leqslant \operatorname{dim} \mathrm{D}^{\mathrm{b}}(\Lambda / r \Lambda)+n
$$

concerning dimensions of the appropriate triangulated categories, in the sense of Rouquier [4]. This inequality gives a way to deduce the finiteness of the dimension of the derived category of $\Lambda$ from that of the derived category of $\Lambda / r \Lambda$. The point is that the ring $\Lambda / r \Lambda$ is "smaller" than $\Lambda$; for example, the Krull dimension of $(\Lambda / r \Lambda)^{c}$

is strictly smaller than that of $\Lambda^{c}$. This approach is predicated on the existence of non-zerodivisors that annihilate Ext-modules. For results in this direction, see [2, Section 7].

\section{Acknowledgments}

We should like to thank the referee for suggestions concerning presentation.

\section{Decompositions}

We deduce the statement in the Introduction from Theorem 2.10 below that concerns derived categories of abelian categories.

Definition 2.1. Let $T$ be a triangulated category, and $\Sigma$ its suspension functor; soon we will focus on the derived category of an abelian category.

Let $C$ be a subcategory (always assumed to be full) of $T$. We write $\operatorname{add}(C)$ for the smallest subcategory of $T$ containing $C$ and closed under finite direct sums, retracts, and shifts. Given a subcategory D of T, the subcategory consisting of objects $E$ that appear in exact triangles of the form

$$
C \rightarrow E \rightarrow D \rightarrow \Sigma C \text { with } C \in \mathrm{C} \text { and } D \in \mathrm{D}
$$

is denoted $\mathrm{C} * \mathrm{D}$. It is convenient to introduce also the following notation:

$$
\mathrm{C} \diamond \mathrm{D}:=\operatorname{add}(\mathrm{C} * \mathrm{D}) \text {. }
$$

It is a consequence of the octahedral axiom that there are equalities

$$
(\mathrm{B} * \mathrm{C}) * \mathrm{D}=\mathrm{B} *(\mathrm{C} * \mathrm{D}) \quad \text { and } \quad(\mathrm{B} \diamond \mathrm{C}) \diamond \mathrm{D}=\mathrm{B} \diamond(\mathrm{C} \diamond \mathrm{D}) .
$$

In particular, we may denote them $B * C * D$ and $B \diamond C \diamond D$, respectively.

Throughout the rest of this section, $R$ will be a commutative ring.

Definition 2.2. An additive category $\mathrm{A}$ is said to be $R$-linear if for each $A$ in $\mathrm{A}$ there are homomorphisms of rings

$$
\eta_{A}: R \rightarrow \operatorname{End}_{\mathrm{A}}(A)
$$

with the property that the action of $R$ on $\operatorname{Hom}_{\mathrm{A}}(A, B)$ induced by $\eta_{A}$ and $\eta_{B}$ coincide, for all $A, B$ in $\mathrm{A}$. Said otherwise, $\operatorname{Hom}_{\mathrm{A}}(A, B)$ is an $R$-module and this structure is compatible with compositions in A. 
Let $\mathrm{A}$ be an $R$-linear Abelian category. The category of complexes over $\mathrm{A}$ inherits an $R$-linear structure, as does the bounded derived category, $D^{\mathrm{b}}(\mathrm{A})$, of $\mathrm{A}$. In either case, the action is compatible with the suspension, in that the morphisms $\Sigma(X \stackrel{r}{\rightarrow} X)$ and $\Sigma X \stackrel{r}{\rightarrow} \Sigma X$ coincide for all $r \in R$ and complexes $X$. What is used repeatedly in the sequel is that for any $r \in R$ and morphism $f: X \rightarrow Y$, in either category, there is an induced commutative square

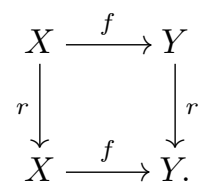

Henceforth, we assume that A has enough projective objects, and write proj A for the corresponding subcategory. For ease of notation, we abbreviate

$$
\begin{aligned}
\mathrm{T} & :=\mathrm{D}^{\mathrm{b}}(\mathrm{A}) \\
\mathrm{P}_{n} & :=\underbrace{\operatorname{proj} \mathrm{A} \diamond \cdots \diamond \operatorname{proj} \mathrm{A}}_{n \text { copies }} \text { for each } n \geqslant 0 .
\end{aligned}
$$

Recall that ghost in T is a morphism $f: X \rightarrow Y$ such that

$$
\operatorname{Hom}_{\mathrm{T}}\left(\Sigma^{n} P, f\right)=0 \quad \text { for all } P \text { in } \operatorname{proj} \mathrm{A} \text { and } n \in \mathbb{Z} \text {. }
$$

In what follows, we write $\mathcal{G}$ for the class of ghosts; it is an ideal in T. For any integer $n$, the ideal $\mathcal{G}^{n}$ consists of morphisms that are $n$-fold compositions of ghosts.

Remark 2.3. For each non-negative integer $n$, one has

$$
\operatorname{Hom}_{\mathrm{T}}(P, g)=0 \quad \text { for all } P \in \mathrm{P}_{n} \text { and } g \in \mathcal{G}^{n} .
$$

This is the well-known Ghost Lemma; for a proof, see, for example, [3, Theorem 3].

Remark 2.4. For each complex $X$ in T and integer $n \geqslant 1$, there is an exact triangle

$$
P \stackrel{p}{\longrightarrow} X \stackrel{q}{\longrightarrow} Y \longrightarrow \Sigma P
$$

with $P$ in $\mathrm{P}_{n}$ and $q$ in $\mathcal{G}^{n}$; one can get this, for instance, from the construction of an Adams resolution of $X$; see [1, Section 4]. When $X$ is in A, such a triangle exists with $\Sigma^{-n} Y$ in A.

Definition 2.5. For $r \in R$, let $\mathrm{T}_{r}$ denote the subcategory of $\mathrm{T}$ consisting of complexes $X$ such that the multiplication morphism $X \stackrel{r}{\rightarrow} X$ is zero in $\mathrm{T}$; in other words, $r$ is in the kernel of the natural map $R \rightarrow \operatorname{End}_{\mathbf{T}}(X)$.

Remark 2.6. Let $r, s$ be elements of $R$. In any exact triangle $X \rightarrow Y \rightarrow Z \rightarrow \Sigma X$ in $\mathrm{T}$, if $X \in \mathrm{T}_{r}$ and $Z \in \mathrm{T}_{s}$, then $Y \in \mathrm{T}_{r s}$ holds.

Indeed, this is a well-known argument (analogous to one for the Ghost Lemma) 
contained in the commutative diagram below:

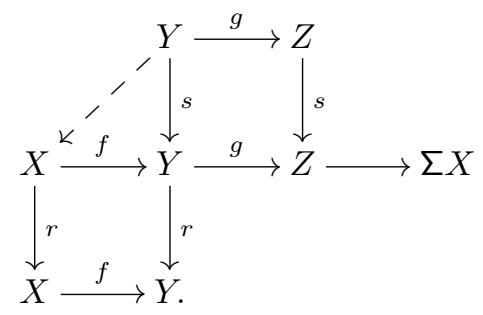

The squares in the diagram are commutative by the definition of the $R$-action on $\mathrm{T}$. The morphism $Y \rightarrow X$ exists because $g s=s g=0$; the second equality holds since $Z$ is in $\mathrm{T}_{s}$. The morphism $Y \stackrel{r s}{\rightarrow} Y$ thus factors as $Y \rightarrow X \stackrel{r}{\rightarrow} X \stackrel{f}{\rightarrow} Y$ and hence is zero, since $X$ is in $\mathrm{T}_{r}$.

In what follows, given a morphism $f: X \rightarrow Y$ of complexes over A, its mapping cone is denoted cone $(f)$; thus

$$
\text { cone }(f)^{n}:=Y^{n} \bigoplus X^{n+1} \quad \text { with differential }\left[\begin{array}{cc}
d^{Y} & f \\
0 & -d^{X}
\end{array}\right]
$$

The canonical exact sequence of complexes

$$
0 \longrightarrow Y \longrightarrow \operatorname{cone}(f) \longrightarrow \Sigma X \longrightarrow 0
$$

gives rise to an exact triangle $X \stackrel{f}{\rightarrow} Y \rightarrow \operatorname{cone}(f) \rightarrow \Sigma X$ in T.

Remark 2.7. For $r \in R$ and complex $X$ over A, set $X / / r:=\operatorname{cone}(X \stackrel{r}{\rightarrow} X)$. Observe that $X / / r$ is in $\mathrm{T}_{r}$, because the map

$$
\left[\begin{array}{ll}
0 & 0 \\
1 & 0
\end{array}\right]: X / / r \longrightarrow X / / r
$$

defines a homotopy between multiplication by $r$ and the zero morphism.

Lemma 2.8. For each subcategory $\mathrm{C}$ of $\mathrm{T}$ and element $r \in R$ there are inclusions

$$
\mathrm{T}_{r} * \mathrm{C} \subseteq \mathrm{C} * \mathrm{~T}_{r^{2}} \quad \text { and } \quad \mathrm{C} * \mathrm{~T}_{r} \subseteq \mathrm{T}_{r^{2}} * \mathrm{C} .
$$

Proof. We verify the first inclusion; the second one can be checked along the same lines.

Fix an $X$ in $\mathrm{T}_{r} * \mathrm{C}$. Thus, there exist $T \in \mathrm{T}_{r}$ and $C \in \mathrm{C}$ and an exact triangle in the top row of the following diagram:

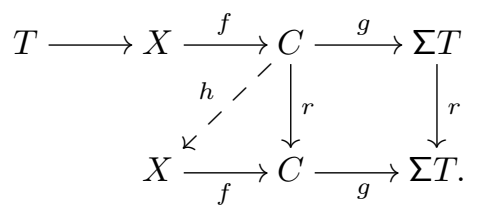

The map $h$ exists because $g r=r g=0$, where the second equality holds because $T$ is in $\mathrm{T}_{r}$. By the octahedral axiom, the factorization $r=f h$ gives rise to an exact 
ANNIHILATION OF COHOMOLOGY AND DECOMPOSITIONS OF DERIVED CATEGORIES235

triangle

$$
T \longrightarrow \operatorname{cone}(h) \longrightarrow C / / r \longrightarrow
$$

It follows from Remarks 2.6 and 2.7 that $r^{2}$ annihilates cone $(h)$. It remains to notice the exact triangle $C \longrightarrow X \longrightarrow$ cone $(h) \rightarrow \Sigma C$.

Definition 2.9. For an element $r \in R$ and an integer $n \geqslant 0$ we consider the following four conditions on the triangulated category $T:=D^{b}(A)$.

$$
\begin{aligned}
& \mathrm{D}_{r, n} \quad \mathrm{~T}=\mathrm{T}_{r} \diamond \mathrm{P}_{n}, \quad \text { and } \quad \mathrm{E}_{r, n} \quad r \operatorname{Ext}_{\mathrm{A}}^{n}(\mathrm{~A}, \mathrm{~A})=0, \\
& \mathrm{D}_{r, n}^{\prime} \quad \mathrm{T}=\mathrm{P}_{n} \diamond \mathrm{T}_{r}, \quad \text { and } \quad \mathrm{G}_{r, n} \quad r \mathcal{G}^{n}=0 \text {. }
\end{aligned}
$$

The statement from the introduction is a consequence of the following theorem.

Theorem 2.10. The following implications hold

$$
\mathrm{D}_{r, n}^{\prime} \Longleftrightarrow \mathrm{D}_{r, n} \Longleftrightarrow \mathrm{G}_{r, n} \Longrightarrow \mathrm{E}_{r, n} \Longrightarrow \mathrm{D}_{r^{3}, 2 n}
$$

Proof. $\left(\mathrm{D}_{r, n}^{\prime} \Rightarrow \mathrm{G}_{r, n}\right)$ : Fix $f: X \rightarrow Y$ to be in $\mathcal{G}^{n}$, and $P \stackrel{p}{\rightarrow} X \stackrel{q}{\rightarrow} T \rightarrow \Sigma P$ the exact triangle provided by the hypothesis. Consider the commutative diagram below where the morphism $X \rightarrow P$ is induced by the fact the $q r=r q=0$, since $T$ is in $\mathrm{T}_{r}$.

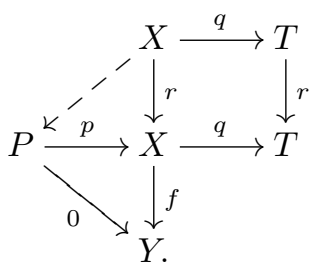

It remains to note that the composition $f p=0$, by Remark 2.3 .

$\left(\mathrm{D}_{r, n} \Rightarrow \mathrm{G}_{r, n}\right)$ can be verified by an argument analogous to the one above.

$\left(\mathrm{G}_{r, n} \Rightarrow \mathrm{D}_{r, n}^{\prime}\right)$ and $\left(\mathrm{G}_{r, n} \Rightarrow \mathrm{D}_{r, n}\right):$ Fix $X$ in $\mathrm{T}$ and $P \stackrel{p}{\longrightarrow} X \stackrel{q}{\longrightarrow} Y \rightarrow \Sigma P$ the exact triangle from Remark 2.4. By hypothesis $r q=0$, so the octahedral axiom applied to the composition $r q$ gives rises to an exact triangle

$$
\Sigma P \longrightarrow Y \bigoplus \Sigma X \longrightarrow Y \| r \rightarrow \Sigma^{2} P
$$

It remains to recall that $Y / / r$ is in $\mathrm{T}_{r}$, by Remark 2.7 , so that property $\mathrm{D}_{r, n}^{\prime}$ holds. Applying the octahedral axiom to the map $q r$, which is also zero, shows that $\mathrm{D}_{r, n}$ holds as well.

$\left(\mathrm{G}_{r, n} \Rightarrow \mathrm{E}_{r, n}\right)$ : This holds because any morphism $f: A \rightarrow \Sigma^{n} B$, with $A, B$ in $\mathrm{A}$, is in $\mathcal{G}^{n}$; see Remarks 2.3 and 2.4 .

$\left(\mathrm{E}_{r, n} \Longrightarrow \mathrm{D}_{r^{3}, 2 n}\right):$ For a start observe that $\mathrm{A} \subseteq \mathrm{T}_{r} \diamond \mathrm{P}_{n}$; this follows by an argument along the lines of the one for $\mathrm{G}_{r, n} \Rightarrow \mathrm{D}_{r, n}^{\prime}$ above. For a complex $X$ over $\mathrm{A}$ let $Z^{*}(X)$ and $B^{*}(X)$ denote the cycles and boundaries of $X$, respectively. There are canonical 
exact triangles

$$
\begin{gathered}
Z^{*}(X) \longrightarrow X \longrightarrow \Sigma B^{*}(X) \longrightarrow \Sigma Z^{*}(X) \\
B^{*}(X) \longrightarrow Z^{*}(X) \longrightarrow H^{*}(X) \longrightarrow \Sigma B^{*}(X) .
\end{gathered}
$$

As $Z^{*}(X)$ and $B^{*}(X)$ are in $\operatorname{add}(\mathrm{A})$, one gets the first of the following chain of inclusions:

$$
\begin{aligned}
\mathrm{T} & \subseteq \mathrm{A} \diamond \mathrm{A} \\
& \subseteq\left(\mathrm{T}_{r} \diamond \mathrm{P}_{n}\right) \diamond\left(\mathrm{T}_{r} \diamond \mathrm{P}_{n}\right) \\
& \subseteq \mathrm{T}_{r} \diamond \mathrm{T}_{r^{2}} \diamond \mathrm{P}_{n} \diamond \mathrm{P}_{n} \\
& \subseteq \mathrm{T}_{r^{3}} \diamond \mathrm{P}_{2 n} .
\end{aligned}
$$

The third inclusion holds by the associativity of $\diamond$ and Lemma 2.8. The last one holds by Remark 2.6 and the definition of the $\mathrm{P}_{n}$. This is the desired implication.

\section{Non-zerodivisors}

Now let $\Lambda$ be a right coherent ring and $r \in \Lambda^{c}$ a non-unit element in the center of $\Lambda$. The homomorphism of rings $\Lambda \rightarrow \Lambda / r \Lambda$ then induces, by restriction of scalars, an exact functor of triangulated categories

$$
\mathrm{D}^{\mathrm{b}}(\Lambda / r \Lambda) \longrightarrow \mathrm{D}^{\mathrm{b}}(\Lambda)
$$

Evidently, its image lies in the subcategory $\mathrm{D}^{\mathrm{b}}(\Lambda)_{r}$.

Lemma 2.11. When $r$ is a non-zerodivisor on $\Lambda$, the functor $\mathrm{D}^{\mathrm{b}}(\Lambda / r \Lambda) \rightarrow \mathrm{D}^{\mathrm{b}}(\Lambda)_{r}$ is dense up to direct summands.

Proof. Since $r$ is a non-zerodivisor on $\Lambda$, the canonical map $\Lambda / / r \rightarrow H^{0}(\Lambda / / r) \cong \Lambda / r \Lambda$ is a quasi-isomorphism in $\mathrm{D}^{\mathrm{b}}(\Lambda)$. This gives rise to an exact triangle

$$
\Lambda \stackrel{r}{\longrightarrow} \Lambda \longrightarrow \Lambda / r \Lambda \longrightarrow \Sigma \Lambda \text {. }
$$

For any $X \in \mathrm{D}^{\mathrm{b}}(\Lambda)_{r}$, applying $X \otimes_{\Lambda}^{\mathrm{L}}$ - yields an exact triangle

$$
X \stackrel{r}{\longrightarrow} X \longrightarrow X \otimes_{\Lambda}^{\mathbf{L}}(\Lambda / r \Lambda) \longrightarrow \Sigma X .
$$

Since the first morphism in this triangle is zero, one gets an isomorphism

$$
X \otimes_{\Lambda}^{\mathbf{L}}(\Lambda / r \Lambda) \cong X \oplus \Sigma X .
$$

Note that $X \otimes_{\Lambda}^{\mathrm{L}}(\Lambda / r \Lambda)$ is in the image of the functor $\mathrm{D}^{\mathrm{b}}(\Lambda / r \Lambda) \rightarrow \mathrm{D}^{\mathrm{b}}(\Lambda)$.

\section{Dimension}

Recall that the dimension of a triangulated category $\mathrm{T}$, denoted $\operatorname{dim} \mathrm{T}$, is the least non-negative integer $d$ for which there exists an object $G$ such that $\{G\}^{(d+1) \diamond}=\mathrm{T}$; see $[\mathbf{4}$, Definition 3.2].

The result below justifies the inequality stated in the introduction. Recall that $\mathcal{G}$ denotes the class of ghosts in $\mathrm{D}^{\mathrm{b}}(\Lambda)$.

Corollary 2.12. Let $\Lambda$ be a right coherent ring. If $r \in \Lambda^{c}$ is a non-zerodivisor on $\Lambda$ and satisfies $r \mathcal{G}^{n}=0$ for some non-negative integer $n$, then there is an inequality

$$
\operatorname{dim} \mathrm{D}^{\mathrm{b}}(\Lambda) \leqslant \operatorname{dim} \mathrm{D}^{\mathrm{b}}(\Lambda / r \Lambda)+n
$$


Proof. Part of the hypothesis is that $\mathrm{D}^{\mathrm{b}}(\Lambda)$ satisfies condition $\mathrm{G}_{r, n}$, in the notation of Theorem 2.10. Keeping in mind Lemma 2.11 and that $\operatorname{proj} \Lambda=\operatorname{add} \Lambda$, op. cit. yields

$$
\mathrm{D}^{\mathrm{b}}(\Lambda)=\mathrm{D}^{\mathrm{b}}(\Lambda / r \Lambda) \diamond\{\Lambda\}^{n \diamond}
$$

We have identified $\mathrm{D}^{\mathrm{b}}(\Lambda / r \Lambda)$ with its image in $\mathrm{D}^{\mathrm{b}}(\Lambda)$. If for some complex $F$ and integer $d$ one has $\mathrm{D}^{\mathrm{b}}(\Lambda / r \Lambda)=\{F\}^{(d+1) \diamond}$, then the equality above yields

$$
\mathrm{D}^{\mathrm{b}}(\Lambda)=\{F \bigoplus \Lambda\}^{(d+n+1) \diamond} .
$$

This implies the desired inequality.

\section{References}

[1] J. Daniel Christensen, Ideals in triangulated categories: phantoms, ghosts and skeleta, Adv. Math. 136 (1998), no. 2, 284-339, DOI 10.1006/aima.1998.1735. MR1626856 (99g:18007)

[2] S. B. Iyengar and R. Takahashi, Annihilation of cohomology and strong generators for module categories, available at arXiv:1404.1476.

[3] G. M. Kelly, Chain maps inducing zero homology maps, Proc. Cambridge Philos. Soc. 61 (1965), 847-854. MR0188273 (32 \#5712)

[4] R. Rouquier, Dimensions of triangulated categories, J. K-Theory 1 (2008), no. 2, 193-256, DOI 10.1017/is007011012jkt010. MR2434186 (2009i:18008)

Srikanth B. Iyengar s.b.iyengar@unl.edu

Department of Mathematics, University of Nebraska, Lincoln, NE 68588-0130, USA

Ryo Takahashi takahashi@math.nagoya-u.ac.jp

Graduate School of Mathematics, Nagoya University, Furocho, Chikusaku, Nagoya 464-8602, Japan 